\title{
Thorough Perioperative Laser Photocoagulation in Prevention of Neovascular Glaucoma after Vitrectomy for Diabetic Macular Edema
}

\author{
Yuka Aoyama-Araki, Fumiyuki Araki, Tomoyasu Shiraya* and Satoshi Kato
}

Department of Ophthalmology, Graduate School of Medicine, The University of Tokyo, Tokyo, Japan

*Corresponding author: Tomoyasu Shiraya, Department of Ophthalmology, Graduate School of Medicine, University of Tokyo, 7-3-1 Hongo, Bunkyo-ku, Tokyo, 113-8655, Japan, Tel: +81-3-3815-5411, Fax: +81-3-3817-0798

\begin{abstract}
Objectives: Neovascular glaucoma (NVG) is a severe complication that may follow pars plana vitrectomy (PPV) for diabetic macular edema (DME). The objective of this study was to evaluate the effectiveness of perioperative fluorescein angiography (FA) and targeted photocoagulation of the non-perfused area (NPA) in preventing the occurrence of postoperative NVG.

Methods: This retrospective, interventional case series study. Vitrectomy for DME was performed by a single surgeon between October 2002 and December 2017. In patients with severe cataracts, FA was performed again after simultaneous phacoemulsification surgery and vitrectomy. When an NPA was detected on FA, targeted photocoagulation was performed on the involved area.

Results: Subjects were 52 patients (56 eyes) who received PPV for DME. Fifty-three eyes had non-proliferative diabetic retinopathy, while three had proliferative diabetic retinopathy. The mean postoperative follow-up period was $38.7 \pm$ 25.2 months. A total of 47 eyes $(83.9 \%)$ eventually underwent pan-retinal photocoagulation. None of the cases developed NVG nor were any other postoperative complications observed.

Conclusions: The current study suggests that perioperative FA and adequate retinal PC for NPA are important strategies for preventing post-operative NVG.
\end{abstract}

\section{Keywords}

Diabetic macula edema, Fluorescein angiography, Neovascular glaucoma, Retinal photocoagulation, Pars plana vitrectomy

\section{Introduction}

Diabetic macula edema (DME) is one of the leading causes of decreased vision in diabetic patients with diabetic retinopathy. According to the $\mathbf{2 0 1 2}$ meta-analysis, $34.6 \%$ of diabetic patients suffered from diabetic retinopathy, and $6.81 \%$ suffered from DME [1]. Laser photocoagulation (PC), intravitreal or sub-Tenon's injection of triamcinolone acetonide, and pars plana vitrectomy (PPV) has been used for treatment of DME. In recent years, the advent of anti-vascular endothelial growth factor (VEGF) therapy has transformed the treatment of DME [2] and has become the first-line treatment for center-involving DME $[3,4]$. In 2014, the Ministry of Health Labour, and Welfare in Japan approved two anti-VEGF agents (ranibizumab; aflibercept) for treatment of DME.

There is a good deal of evidence suggesting that PPV also effectively restores retinal function and significantly decreases DME [5-7]. According to the recent trend following introduction of anti-VEGF agents, candidates for PPV include those with vitreomacular traction and those with an epiretinal membrane detected by optical coherence tomography in current clinical practice [5].

Pars plana vitrectomy seems effective in DME but is associated with certain complications, such as cataract, retinal detachment [8]. Moreover, vitrectomized eyes often have postoperative progression of anterior segment neovascularization, which can lead to neovascular glaucoma (NVG) [9]. Among these NVG is a severe com- 
plication following PPV, which can occur at any point in time during the observation period and can potentially lead to blindness. In the long-term follow-up data, the reported rate of NVG was 3.9\% [10], while another report placed it at $4.6 \%$ [11], suggesting that it is important to evaluate ischemic states and monitor patients on a monthly basis in the clinic [5].

The present study investigated retrospectively whether post-operatively NVG could be prevented by strictly targeted retinal photocoagulation (TRP) when non-perfused areas (NPA) were detected on fluorescein angiography (FA).

\section{Methods}

This retrospective, interventional case series study was performed from October 2002 to December 2017 at The University of Tokyo Hospital (Tokyo, Japan) and approved by the Ethical Committee of Tokyo University Graduate School of Medicine (\#2217). All patients underwent a comprehensive ophthalmic examination, and informed consent was obtained from each of the patients after they were provided sufficient information on the planned procedures. All subjects were screened for NVG before and after surgery with thorough slitlamp examination of the iris/pupillary margin, gonioscopy of the anterior chamber angle, and measurement of intraocular pressure. Neovascular glaucoma was defined as the presence of vessels at the pupillary margin or in the anterior-chamber angle.

Vitrectomy was selected as the best treatment for cases in which central macular thickness remained at $300 \mu \mathrm{m}$ or more (measured using optical coherence tomography) after a sub-Tenon's steroid injection administration, for cases of apparent vitreomacular traction or an epiretinal membrane detected by optical coherence tomography. After the launch of anti-VEGF agents, vitrectomy was selected as the best treatment when anti-VEGF therapy was contraindicated because of risk, economic problems, or a history of a cerebral infarction or cardiovascular event. The perioperative period was defined as the time encompassing 1 year before and 1 year after surgery.

In the included cases, FA was performed within 1 year of surgery, in cases with unclear images due to an intervening translucent opacity, FA was performed within 1 year after surgery. Eyes with a distinct and extensive capillary NPA ( $>5$-disc areas on the major axis on FA) underwent TRP and eyes with retinal neovascularization (identified with FA) underwent pan retinal photocoagulation (PRP), and single-spot photocoagulation was performed in all cases requiring PRP. Patients with a history of NVG or anti-VEGF therapy, eyes with a vitreous hemorrhage that prevented DME evaluation were excluded from analysis.

A standard three-port PPV using a 20G or 23G system under local anesthesia was performed by a single sur-
Table 1: Baseline clinical characteristics of the patients with diabetic macular edema.

\begin{tabular}{|l|l|}
\hline Characteristics & \\
\hline No. of eyes & 56 \\
\hline Sex (male/female) & $29 / 23$ \\
\hline Mean age mean \pm SD (range) & $62.3 \pm 9.24$ (42-84) \\
\hline $\begin{array}{l}\text { Stage of diabetic retinopathy, } \mathrm{n}(\%) \\
\text { Non-PDR*, } \mathrm{n}(\%)\end{array}$ \\
\hline \multicolumn{1}{|c|}{ PDR, $\mathrm{n}(\%)$} & $53(94.6)$ \\
\hline Phakic lens status, $\mathrm{n}(\%)$ & $3(5.4)$ \\
\hline Case of PVD incomplete, $\mathrm{n}(\%)$ & $45(80.4)$ \\
\hline
\end{tabular}

"non-PDR = All eyes included in this study had severe NPDR (eyes with mild or moderate NPDR were not included). $\mathrm{n}=$ number of eyes, SD = Standard Deviation, $\mathrm{PDR}=$ Proliferative Diabetic Retinopathy, PVD = Posterior Vitreous Detachment.

Table 2: Laser treatment during observation period $(n=56)$.

\begin{tabular}{|l|l|}
\hline & $\mathrm{n}(\%)$ \\
\hline Preoperative & $52(92.9)$ \\
\hline Focal photocoagulation & $9(16.0)$ \\
\hline Pan-retinal photocoagulation & $43(76.8)$ \\
\hline Intraoperative & $10(17.9)$ \\
\hline Postoperative & $6(10.7)$ \\
\hline No photocoagulation & $1(1.8)$ \\
\hline
\end{tabular}

$\mathrm{n}=$ number of eyes.

geon (K.S.). All phakic eyes underwent lens phacoemulsification and intraocular lens implantation. After core vitrectomy, a posterior vitreous detachment was induced using triamcinolone acetonide in cases in which it had not occurred. The internal limiting membrane was not removed in any case. Intraoperative-PC was added in cases with vitreous hemorrhage or with insufficient pre-operative laser treatment to the peripheral retina.

\section{Results}

Subjects were 52 patients who received PPV for $\mathrm{DME}$, and Table 1 shows the baseline clinical characteristics of the patients. The mean postoperative follow-up period was $38.7 \pm 25.2$ months. All subjects had an intraocular pressure of $21 \mathrm{mmHg}$ or less both before and after surgery. Table 2 shows the timing of PC; a total of 47 eyes (83.9\%) eventually underwent PRP. None of the cases developed NVG nor were any other postoperative complications, such as expulsive hemorrhage, retinal detachment, and postoperative endophthalmitis, observed.

\section{Discussion}

Anti-VEGF agents have become the first line therapy for DME in recent years. However, vitrectomy is still indicated in eyes with vitreomacular traction and/or an epiretinal membrane detected by optical coherence tomography [5]. Therefore, vitrectomy remains an important treatment option for eyes with DME in clinical practice. Postoperative NVG is a serious complication associated with vitrectomy that can cause blindness. Unfortunately, there have been no reports that focus on postoperative NVG prevention. In our study, none of subjects developed NVG after vitrectomy, perhaps 
because of thorough PC in the perioperative period. In addition, we also think it important to perform a postoperative FA in cases where it could not be conducted preoperatively or where additional intraoperative-PC was performed. Previous reports have mentioned that neovascularization of the iris usually presents within 1-2 months postoperatively [11]. Although, we reviewed FA findings within 1 year of surgery, it is better to perform FA within 1 month of vitrectomy so that additional PC can be performed if necessary. Although a thorough PC for retinal ischemia is necessary to prevent NVG, over-treatment that could cause visual-field defects or aggravation of DME should be avoided. The Early Treatment Diabetic Retinopathy Study Group reported that a mild scatter PC treatment (400-600 laser shots) was significantly more effective in preventing narrowing of the visual field compared with full scatter treatment with 1200-1600 shots [12]. Moreover, we have previously reported that the burn expansion ratio after $P C$ is larger in spots created with intraoperative-PC than in those created with a transpupillary PC [13]. It is clear from these findings that excessive laser treatment with a large number of laser shots should be avoided, and $P C$ is preferable before surgery in cases where NPA is confirmed preoperatively.

In summary, the current study suggests that perioperative FA and adequate retinal PC for NPA are important strategies for preventing post-operative NVG.

\section{Conflict of Interest}

The authors declare that they have no conflict of interest.

\section{References}

1. Yau JW, Rogers SL, Kawasaki R, Lamoureux EL, Kowalski JW, et al. (2012) Global prevalence and major risk factors of diabetic retinopathy. Diabetes Care 35: 556-564.

2. Stewart MW (2014) Anti-VEGF therapy for diabetic macular edema. Curr Diab Rep 14: 510.
3. Bandello F, Cunha-Vaz J, Chong NV, Lang GE, Massin P, et al. (2012) New approaches for the treatment of diabetic macular oedema: recommendations by an expert panel. Eye (Lond) 26: 485-493.

4. Mitchell $P$, Wong TY, Diabetic Macular Edema Treatment Guideline Working Group (2014) Management paradigms for diabetic macular edema. Am J Ophthalmol 157: 505513.

5. Terasaki H, Ogura Y, Kitano S, Sakamoto T, Murata T, et al. (2018) Management of diabetic macular edema in Japan: a review and expert opinion. Jpn J Ophthalmol 62: 1-23.

6. Lewis H, Abrams GW, Blumenkranz MS, Campo RV (1992) Vitrectomy for diabetic macular traction and edema associated with posterior hyaloidal traction. Ophthalmology 99: 753-759.

7. Yamamoto S, Yamamoto T, Ogata K, Hoshino A, Sato E, et al. (2004) Morphological and functional changes of the macula after vitrectomy and creation of posterior vitreous detachment in eyes with diabetic macular edema. Doc Ophthalmol 109: 249-253.

8. Diabetic Retinopathy Clinical Research Network Writing Committee, Haller JA, Qin H, Apte RS, Beck RR, et al. (2010) Vitrectomy outcomes in eyes with diabetic macular edema and vitreomacular traction. Ophthalmology 117 : 1087-1093.

9. Goto A, Inatani M, Inoue T, Awai-Kasaoka N, Takihara Y, et al. (2013) Frequency and risk factors for neovascular glaucoma after vitrectomy in eyes with proliferative diabetic retinopathy. J Glaucoma 22: 572-576.

10. Kumagai K, Furukawa M, Ogino N, Larson E, Iwaki M, et al. (2009) Long-term follow-up of vitrectomy for diffuse nontractional diabetic macular edema. Retina 29: 464-472.

11. Yamamoto T, Hitani K, Tsukahara I, Yamamoto S, Kawasaki R, et al. (2003) Early postoperative retinal thickness changes and complications after vitrectomy for diabetic macular edema. Am J Ophthalmol 135: 14-19.

12. (1991) Early photocoagulation for diabetic retinopathy. ETDRS report number 9. Ophthalmology 98: 766-785.

13. Shiraya T, Kato S, Araki F, Yamaguchi T, Kaiya T (2015) Comparison of burn sizes resulting from photocoagulation using a transpupillary laser and an endolaser. Acta Ophthalmol 93: e595-e596. 